\title{
Airway inflammation in patients with chronic non-asthmatic cough
}

\author{
Marcin Grabowski, ${ }^{1,2}$ Sven Seys, ${ }^{2,3}$ Ann Decraene, ${ }^{3}$ Ahmad Kasran, ${ }^{2}$ Ellen Dilissen, ${ }^{2}$ \\ Wojciech Barg, ${ }^{1}$ Wojciech Medrala, ${ }^{4,5}$ Lieven J Dupont, ${ }^{3}$ Bernard Panaszek, ${ }^{4}$ \\ Dominique M A Bullens,
}

\begin{abstract}
- Additional supplementary files are published online only. To view these files please visit the journal online (http://dx. doi.org/10.1136/thoraxjnl2012-201895).

${ }^{1}$ Department of Physiology, Wroclaw Medical University, Wroclaw, Poland

${ }^{2}$ Clinical Immunology, Department of Microbiology and Immunology, KULeuven, Leuven, Belgium

${ }^{3}$ Pneumology, Department of Clinical and Experimental

Medicine, KULeuven, Leuven, Belgium

${ }^{4}$ Department of Internal Medicine, Geriatrics and Allergology, Wroclaw Medical University, Wroclaw, Poland ${ }^{5}$ Department of Clinical Research, Medical School of Legnica, Legnica, Poland

${ }^{6}$ Pediatric Immunology, Department of Microbiology and Immunology, KULeuven, Leuven, Belgium

${ }^{7}$ Clinical Pediatrics, UZ Leuven, Leuven, Belgium
\end{abstract}

\section{Correspondence to} Dr Marcin Grabowski, Department of Physiology, Wroclaw Medical University, ul. Chalubinskiego 10, Wroclaw 50-368, Poland;

marcingrabow@02.pl

Received 12 March 2012 Revised 2 September 2012 Accepted 21 September 2012 Published Online First 23 October 2012

To cite: Grabowski M, Seys $S$, Decraene $A$, et al. Thorax 2013;68:125-130.

\section{ABSTRACT}

Introduction Chronic non-asthmatic cough (CC) is a clinical challenge and underlying pathophysiological mechanisms remain still not completely understood. One of the most common comorbidities in CC is gastrooesophageal reflux disease (GORD). Airway epithelium damage can contribute to airway inflammation in CC. Aims We studied airway inflammation in patients with CC compared to healthy controls. Patients with GORD were treated with proton pump inhibitors (PPI) and cough response to PPI was evaluated.

Patients and methods Sputum was induced in 41 adults with CC and 20 healthy non-smokers who were age and sex matched. We compared sputum differential cell count by cytospin and cytokine and chemokine production at the mRNA and/or protein levels by real-time (RT)-PCR and cytokine bead array (CBA), between patients with $\mathrm{CC}$ and healthy subjects. Furthermore we studied airway inflammation in patients with different comorbidities. Results No differences in sputum differential cell counts were observed between patients with $\mathrm{CC}$ and healthy subjects. Sputum monocyte chemoattractant protein-1 (MCP-1) protein levels were significantly higher in patients when compared to controls. Thymic stromal lymphopoietin (TSLP) mRNA was significantly more often expressed in sputum of patients with $\mathrm{CC}$ than from healthy controls. Sputum transforming growth factor (TGF)- $\beta$ levels did not differ between patients and controls, but were significantly lower in the PPI responders compared to the nonresponders; $p=0.047$. There is no evidence for impaired $T$ helper cell (Th)1/Th2/Th17 balance in CC. Patients with reflux oesophagitis (RO) have significantly more sputum eosinophils than patients without RO.

Conclusions $\mathrm{CC}$ is a condition presenting with different disease phenotypes. High sputum MCP-1 levels are present in a large group of patients with $\mathrm{CC}$ and a majority of these patients with CC have increased sputum TSLP levels, most likely produced by damaged airway epithelial cells.

\section{INTRODUCTION}

Chronic cough (CC), which is defined as cough lasting for more than 8 weeks, ${ }^{1}$ is a serious socioeconomical problem and often a challenge for doctors. Most often cough is one of the symptoms in subjects with asthma, chronic obstructive pulmonary disease (COPD), post nasal drip syndrome or chronic infection, but cough might also be associated with gastro-oesophageal reflux disease (GORD). ${ }^{2}$ Whereas infections, allergy or GORD themselves can relatively easily be diagnosed and
Key messages

Patients with chronic non-asthmatic cough have hallmarks of mild airway inflammation, probably related to epithelial damage: higher sputum MCP-1 levels and increased expression of sputum TSLP mRNA compared to controls. However, differences in airway inflammation depending on the comorbidity exist. Therefore, understanding the different chronic cough phenotypes can lead to optimal treatment.

treated, the causal treatment of $\mathrm{CC}$ as a presenting symptom often remains unsuccessful. ${ }^{3}$ The underlying airway inflammation in patients with $\mathrm{CC}$ is still poorly characterised. In GORD-associated CC, the 'reflex' theory emphasises the role of neuronal connections between oesophagus and airways and, as a result of oesophagus stimulation by gastric content, the release of neuropeptides, leading to neuroimmune airway inflammation. ${ }^{4}$ However, the gastrooesophageal 'reflux' theory states that gastric content reaching the pharynx, can also lead to microaspiration into the airways. ${ }^{5}$ Moreover, in animal models of microaspiration a predominant role for $\mathrm{T}$ helper 2 (Th2) lymphocytes in airway inflammation caused by microaspiration has been documented. ${ }^{6}$ However, we recently revealed that patients with $\mathrm{CC}$ and healthy subjects have similar sputum pepsin and biliary acid levels, arguing against a major role for microaspiration of gastric content into the airways in the pathophysiology of CC. ${ }^{7}$

The involvement of the epithelium in the pathogenesis of airway disease is increasingly acknowledged. Activated epithelial cells are potent sources of haematopoietic cytokines such as granulocyte (macrophage) colony stimulating factor (G(M)-CSF), and chemokines such as regulated upon activation normal $\mathrm{T}$ cell expressed and secreted (RANTES), thymic stromal lymphopoietin (TSLP), eotaxin-1, monokine induced by interferon- $\gamma$, interferon-inducible protein-10 (IP-10), interleukin (IL)-8 and monocyte chemoattractant protein-1 (MCP-1). ${ }^{89}$

Foregoing studies on airway inflammation in chronic unexplained cough are often heterogeneous, thus difficult to compare. Our study aims to characterise airway inflammation in patients with $\mathrm{CC}$, in whom asthma, COPD and infections where 
excluded. We used induced sputum as a well validated technique widely used to assess and monitor airway inflammation. ${ }^{10}$ In this study we compared markers of airway inflammation present in induced sputum obtained from patients with $\mathrm{CC}$ and healthy controls (HC) at the mRNA and at the protein level as well as in different CC phenotypes (atopic vs non-atopic, with reflux oesophagitis (RO) or idiopathic CC (ICC)). We studied the role of $\mathrm{T}$ cell cytokines, as well as chemokines and cytokines released by epithelial cells, probably responsible for attracting other inflammatory cells (eg, eosinophils and neutrophils) to the airways in CC.

\section{MATERIALS AND METHODS}

\section{Study subjects}

Patients who were referred due to $\mathrm{CC}$ to the Department of Internal Medicine, Geriatrics and Allergology of Wroclaw Medical University between December 2009 and February 2010 were enrolled in the study, and underwent a diagnostic protocol adapted from European Respiratory Society (ERS) guidelines. ${ }^{1}$ Adult patients (18-75 years), non-smokers or past smokers (pack years $<5$ ) with CC were included. Patients have not used inhaled steroids or angiotensin-converting enzyme inhibitors for at least 6 weeks before investigation and were allowed to use proton pump inhibitor (PPI) treatment at the time of sputum induction.

At the time of sputum induction 12 patients were already treated by PPI (omeprazole $40 \mathrm{mg}$ or pantoprazole $80 \mathrm{mg}$ daily) for at least 2 weeks (see table 1). After sputum induction all patients were uniformly treated with omeprazole $40 \mathrm{mg}$ per day $(n=39)$. The response to PPI treatment was assessed 4 weeks later. A positive response to PPI treatment was defined as a partial or complete resolution of cough $(n=15)$. When cough did not improve sufficiently after at least 4 weeks treatment with PPI, patients were scored to have a negative response to PPI $(n=12)$. In two subjects it was not possible to record the impact of PPI treatment on CC, so they were not included in table 1.

Patients with asthma (also 'cough variant asthma'), chronic sinusitis, tuberculosis and chlamydial or mycoplasmal infections were identified and excluded. In total, 41 patients were included (see table 2).

The control group consisted of 20 healthy volunteers, age and sex matched, who were non-smokers with negative allergy history, no clinical symptoms of upper or lower airway disease and without GORD-related symptoms. As they had negative history of allergy, no skin prick tests were performed in this group (table 2).

Table 1 Occurrence of study subjects according to the ongoing proton pump inhibitor (PPI) treatment at the time of sputum induction and distribution of patients treated with PPI at the time of sputum induction and their response to PPI

\begin{tabular}{|c|c|c|c|}
\hline \multirow[b]{2}{*}{ Number of patients } & \multicolumn{2}{|c|}{$\begin{array}{l}\text { PPI response (after } \\
4 \text { weeks treatment) }\end{array}$} & \multirow[b]{2}{*}{ Total } \\
\hline & Positive & Negative & \\
\hline \multicolumn{4}{|c|}{$\mathrm{PPI}$ at the time of sputum induction ( $>2$ weeks): } \\
\hline Yes & 9 & 3 & 12 \\
\hline No & 15 & 12 & 27 \\
\hline Total & 24 & 15 & \\
\hline
\end{tabular}

The study was approved by the local ethical committee of Wroclaw Medical University. Informed consent was obtained from all study subjects.

\section{Diagnostic procedures}

Gastroscopy was performed in 41 patients. One doctor performed all gastroscopies using GIF-Q145 (Olympus) after suitable local anaesthesia. The diagnosis of endoscopic changes (RO) was based on the Los Angeles classification. ${ }^{11}$ Exhaled NO (eNO) was measured by a chemiluminescence analyser (NIOX Flex; Aerocrine AB, Stockholm, Sweden).

Atopy was diagnosed by skin prick testing (SPT) for common environmental allergens (house dust mites: Dermatophagoides pteronyssinus, Dermatophagoides farinae, cat, dog, tree, grass and weed pollen, and moulds: Alternaria spp. and Cladosporium spp.). SPT were performed according to the standard procedure using reagents supplied by Allergopharma (Germany). Impact of cough on quality of life (QoL) was tested by Polish-translated (non-validated) Leicester Cough Questionnaire (LCQ). ${ }^{12}$

\section{Sputum induction and processing}

Induction of sputum, cytospin preparation and sputum cytokine mRNA measurements were performed as previously described. ${ }^{13-17}$ All mRNA values presented in the text were normalised to $\beta$-actin and multiplied by $10^{4}$. We previously described that sputum CD3 $\gamma$ mRNA levels reflect airway lymphocyte counts. ${ }^{15}$ We therefore studied sputum CD3 $\gamma$ mRNA levels in patients with CC and HC.

\section{Chemokine cytometric bead array measurements}

Levels of IL-8 (CXCL8), monocyte chemotactic protein-1 (MCP-1; CCL2), macrophage inflammatory protein-1 $\alpha$ (MIP-1 $\alpha$; CCL3), interferon inducible protein (IP-10; CXCL10) and transforming growth factor (TGF)- $\beta$ in sputum supernatants were measured by cytometric bead array (CBA) flex sets (BD Bioscience-PharMingen, San Diego, California, USA). The lowest level of accurate detection for this assay was for IL-8: $1.2 \mathrm{pg} / \mathrm{ml}$, MCP-1: $1.3 \mathrm{pg} / \mathrm{ml}, \mathrm{MIP}-1 \alpha$ : $0.2 \mathrm{pg} / \mathrm{ml}$, and IP-10: $0.5 \mathrm{pg} / \mathrm{ml}$, TGF- $\beta: 14.9 \mathrm{pg} / \mathrm{ml}$.

\section{Statistical analysis}

Normality was tested by Shapiro-Wilk's W test and Student $t$ test was used to compare two groups with normal distribution. Differences between the groups with non-Gaussian distribution

Table 2 Subject characteristics

\begin{tabular}{|c|c|c|c|}
\hline $\begin{array}{l}\text { Parameter (median, } \\
\text { IQR) }\end{array}$ & Chronic cough & $\begin{array}{l}\text { Healthy } \\
\text { controls }\end{array}$ & $\mathrm{p}$ Value \\
\hline Age, years & $45.0(29-57)$ & $40.0(36-60)$ & 0.74 \\
\hline Gender, M/F & $30 / 11$ & $13 / 7$ & 0.72 \\
\hline $\begin{array}{l}\text { Time of cough duration, } \\
\text { years }\end{array}$ & $2.0(0.8-5.0)$ & NA & - \\
\hline $\begin{array}{l}\mathrm{FEV}_{1} \text {, percentage } \\
\text { predicted }\end{array}$ & $106.7(100.0-117.25)$ & - & - \\
\hline FVC, percentage predicted & $106.8(98.2-111.3)$ & - & - \\
\hline Tiffeneau, $\mathrm{FEV}_{1} / \mathrm{FVC}$ & $103.0(98.4-108.0)$ & - & - \\
\hline eNO, ppb & $13.0(9.9-16.3)$ & $16.2(12.3-17.6)$ & 0.14 \\
\hline Atopy, yes/no & $8 / 33$ & NA & \\
\hline
\end{tabular}


Figure 1 Concentration of monocyte chemoattractant protein-1 (MCP-1) in induced sputum of patients with chronic cough and the control group (healthy controls), and thymic stromal lymphopoietin (TSLP) representation in sputum was measured in 41 patients and 20 controls. Supernatants were stored and cells were isolated. cDNA was extracted and transcribed to mRNA. MCP-1 levels were measured by cytokine bead array in the sputum supernatants. Median values and IQRs are represented (A). TSLP mRNA levels were measured by reverse transcription (RT)-PCR and normalised to $\beta$-actin and the number of individuals expressing TSLP mRNA in both groups is depicted (B).
A

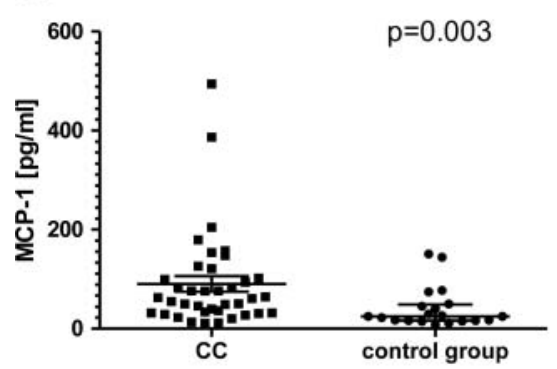

B

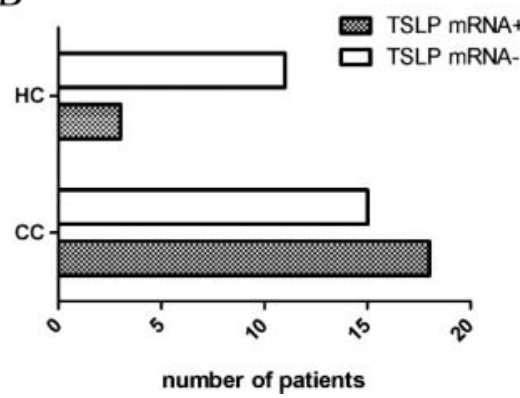

were estimated by the non-parametric Mann-Whitney test for two groups or Kruskal-Wallis test where appropriate. Correlations were studied by Spearman or Pearson (if normally distributed) coefficients (r). Fisher's exact test was used to compare categorical variables between groups. A probability of $\mathrm{p}<0.05$ was regarded as significant (STATISTICA V.8.0, StatSoft, Poland).

\section{RESULTS}

Differential cell counts and expression of sputum epithelial cell cytokines in patients with CC and healthy subjects

We first studied airway differential cell counts in patients with $\mathrm{CC}$ and healthy subjects by means of sputum cytospins. No differences in total cell count or in relative or absolute numbers of epithelial cells, neutrophils, macrophages, eosinophils or lymphocytes could be observed between both groups (data not shown).

We then hypothesised that epithelial cells triggered in CC could lead to epithelial cell cytokine and/or chemokine production in the airways of patients with CC. We therefore measured the expression of sputum epithelial cell cytokines and chemokines in patients with $\mathrm{CC}$ in comparison to healthy control subjects.

As shown in figure 1A, sputum MCP-1 protein concentrations were significantly higher in patients when compared to controls. Along the same lines, sputum IL-8 mRNA (median in CC: 38093 (IQR: $20670-57376$ ) versus median in HC: 23413 (IQR: 18 398-38 722) mRNA copies, $p=0.19$ ) and protein levels (median in CC: 737 (IQR: 480-1306) vs median in HC: 528 (IQR: 366-1145) pg/ml, p=0.29) (which correlated significantly with each other in the patient group, $\mathrm{r}=0.5$, $\mathrm{p}=0.001$, data not shown), as well as sputum IL-6 mRNA levels (median in CC: 368 (IQR: 167-632) vs median in HC: 258 (IQR: 148-588) mRNA copies, $\mathrm{p}=0.21$ ) tended to be higher in the patient group than in the control group. Moreover TSLP mRNA was significantly more often expressed in sputum samples of patients with CC than from healthy control subjects. Sputum TSLP mRNA was present in $54.5 \%$ of the patients with $\mathrm{CC}$ versus $21.4 \%$ of the healthy control subjects $(p=0.04$, figure $1 B)$. The relative risk in patients in whom sputum TSLP mRNA was detectable, was significantly increased to 1.5 (95\% CI 1.023 to 2.157$)$.

We found no differences in sputum IP-10 and MIP- $1 \alpha$ protein levels or sputum eotaxin-1 mRNA levels between both groups (data not shown).

We then studied whether epithelial cell chemokine presence in airways might be related to neutrophil attraction in patients with CC. As shown in figure 2A,B, sputum MCP-1 and IL-8 protein levels correlated significantly with sputum absolute neutrophil counts.

\section{Sputum T cell cytokine expression in patients} with CC and healthy subjects

Similar to our observations regarding lymphocyte counts on cytospin, no difference in sputum CD $\gamma \gamma$ mRNA expression
Figure 2 Correlation between absolute neutrophil count and concentration of monocyte chemoattractant protein-1 (MCP-1) (A) and interleukin (IL)-8 (B) protein expression in the supernatants from patients with chronic cough. Sputum was induced. Cytospins were prepared and the percentage of neutrophils was measured. Absolute neutrophil counts were calculated. MCP-1 and IL-8 levels in sputum supernatants were measured by cytokine bead array. The correlation between sputum absolute neutrophil cell counts and sputum MCP-1 (A) and IL-8 (B) levels is shown.
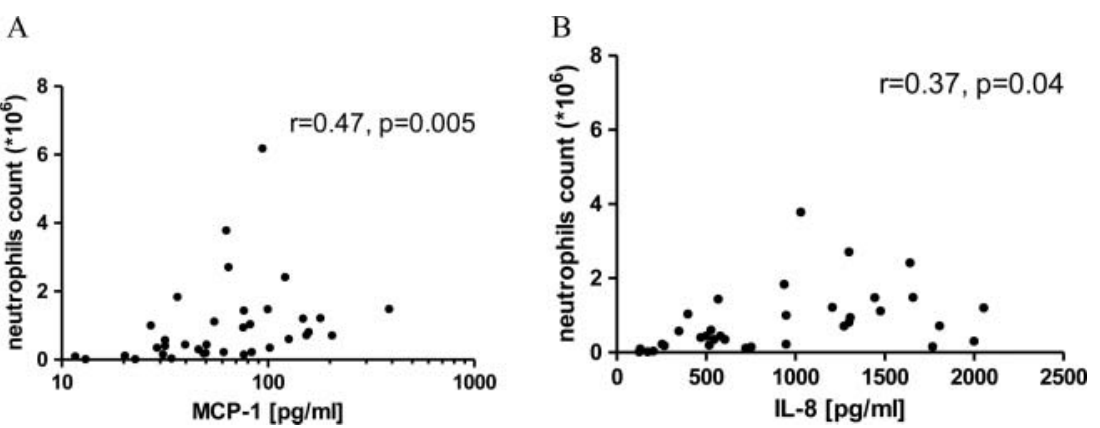
Table 3 Sputum T cell cytokine mRNA expression normalised to $\beta$-actin (multiplied by $10^{4}$ ) in patients with chronic cough and control groups

\begin{tabular}{lccl}
\hline & Chronic cough & Healthy subjects & $p$ Value \\
\hline Th1 (IFN $\gamma)$ & $0.68(0.37-1.27)$ & $0.91(0.37-2.60)$ & 0.48 \\
(IL-12p35) & $0.87(0.00-1.44)$ & $0.56(0.00-1.31)$ & 0.32 \\
(IL-12p40) & $0.00(0.00-0.16)$ & $0.19(0.00-2.19)$ & 0.22 \\
Th2 (IL-5) & $451.01(134.52-1852.20)$ & $729.07(213.21-4902.94)$ & 0.48 \\
Th2/Treg (IL-10) & $2.89(1.58-5.26)$ & $4.46(1.22-9.13)$ & 0.54 \\
Th17 (IL-17A) & $27.92(9.39-151.51)$ & $549.89(18.66-465.69)$ & 0.45 \\
(IL-23p19) & $214.56(53.53-726.96)$ & $341.56(119.70-2039.74)$ & 0.14 \\
\hline
\end{tabular}

The values are represented as medians and IQR (median (p25 to p75)).

IFN, interferon; IL, interleukin; Th, T helper cells; Treg, T regulatory cells.

between patients with CC and control individuals was found (median expression in patient group: 27.6 (IQR: 22.4-48.4), median control group: 33.7 (IQR: 19.6-74.0), $\mathrm{p}=0.50$; data not shown). Sputum RANTES mRNA levels (as chemokine responsible for lymphocyte and monocyte/macrophages migration and maturation) tended to be higher in patients with CC compared to HC (median in CC group: 110.5 (IQR: 61.7-169.9) vs median in HC: 73.7 (IQR: 0.0-119.0) in control group; $\mathrm{p}=0.19$, data not shown). We furthermore studied the expression of sputum T cell (Th1/Th2/Th17) cytokine mRNA levels in patients with $\mathrm{CC}$ and healthy subjects. Results were similar in both groups (table 3 ).

\section{Differences in airway inflammation between patients with CC, depending on comorbidities}

We finally studied whether differences in cellular inflammation and/or sputum cytokine/chemokine expression could be observed among the patients with CC themselves, depending on probable comorbidities.

We first studied differences between atopic and non-atopic patients with CC. Atopy was defined as having at least one positive result in skin prick tests. Eight patients had at least one positive skin prick test (19.5\%). No differences in airway cellularity, FeNO, or sputum cytokine/chemokine expression levels between atopic and non-atopic patients with CC were found (data not shown).

We then compared airway inflammation in patients with RO $(\mathrm{RO}+, \mathrm{n}=27)$ and those without $\mathrm{RO}(\mathrm{RO}-, \mathrm{n}=10)$. As indicated in figure 3, relative and absolute eosinophil counts were higher in patients with $\mathrm{RO}$ in comparison to those without RO. However, no significant differences in sputum IL-5 (median in RO+: 630.8 (IQR: 128.2-1892.3) vs median in RO-: 584.5 (247.8-1847.2) mRNA copies, $\mathrm{p}=0.89)$ and/or eotaxin-1 mRNA levels (median in RO+: 0.0 (IQR: 0.0-1.2) versus median in RO-: 0.1 (IQR: 0.0-1.9), p=0.63) (although both are eosinophil attractants), nor in interferon (IFN) $\gamma$ mRNA levels (median RO+: 0.6 (IQR: $0.3-1.2$ vs median in RO-: 0.7 (IQR: $0.6-1.4), p=0.27$ ) between patients with $\mathrm{CC}$ with RO and patients with $\mathrm{CC}$ without $\mathrm{RO}$ could be observed. A total of 17 out of 27 patients with RO reported that their cough responded to PPI treatment compared with $5 / 10$ without RO who were PPI responders. We concluded that the presence of reflux oesophagitis is not an independent predictive factor for positive response of cough symptoms to PPI treatment (OR: $1.3 ; 95 \%$ CI 0.3 to 5.2 ).

Other sputum cell subpopulations were similar between these two groups (data not shown).

Finally, we also studied the sputum cytokine and chemokine expression in the airways from patients with CC related to treatment with PPI and the response to this treatment in terms of CC resolution (see table 1). The PPI non-responders were classified as having 'ICC'. We could not find any differences in age, gender distribution, severity of cough, lung function, PC20 or eNO levels between patients 'on IPP' and 'off IPP' or between ICC and PPI responders (data not shown). PPI non-responders, however, had the tendency to report more severe cough in the LCQ, median in ICC: 66.0 (IQR: 56.9-83.0) versus median in PPI responders: 79.0 (IQR: $59.0-100.0$ ) than responders, $\mathrm{p}=0.27$.

TGF- $\beta$ sputum protein levels were significantly lower in the PPI responders compared to the non-responders group: medians $105.8 \mathrm{ng} / \mathrm{ml}$ (IQR: 61.0-170.2) versus $177.6 \mathrm{ng} / \mathrm{ml}$ (IQR: $119.3-274.0$ ); $p=0.047$ (figure 4A). TGF- $\beta$ sputum protein levels correlated with sputum absolute eosinophil counts (figure 4B). We found no correlation between sputum TGF- $\beta$ protein levels and age of the patient, cough duration or cough severity (data not shown).

\section{DISCUSSION}

In this study we defined airway inflammation in patients with $\mathrm{CC}$ by means of the non-invasive method of sputum induction.

Bronchial epithelium is highlighted as a key site for the regulation of chemokine expression in health and disease conditions. ${ }^{18}$ Detachment of human bronchial epithelial cells from their basal membrane or slight stretch of the epithelial cells should be shown to induce expression and release of chemokines, such as IL- ${ }^{19}{ }^{20}$ and to be responsible for subepithelial fibrosis by increased expression of TGF- $\beta .^{21}$ Thus, we hypothesised that upon epithelial triggering in $\mathrm{CC}$ the epithelium might start to produce chemokines. The triggering factor in CC can, among other, be vagal nerve stimulation and mediator release caused as reflex reaction upon gastro-oesophageal reflux, or direct contact of the epithelial cells with microaspirates upon this reflux, as well as the repetitive mechanical effects of coughing bouts on the airway epithelium. We observed a significantly
Figure 3 Presence of eosinophils in induced sputum of patients with reflux oesophagitis (RO+) and without RO (RO-) Sputum was induced and cytospins were made. The presence of RO was studied by gastroscopy. Eosinophils on cytospins were counted and the relative $(A)$ and absolute $(B)$ number of eosinophils in sputum samples in each group is represented. Horizontal bars represent median and IQR.
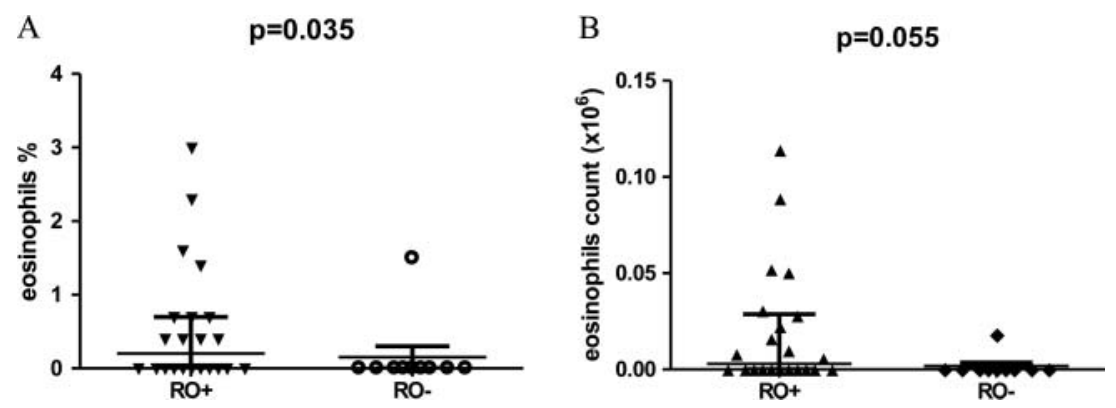
Figure 4 Levels of transforming growth factor (TGF)- $\beta$ in patients with idiopathic chronic cough (ICC) compared to patients in whom cough responded well to proton pump inhibitors treatment. TGF- $\beta$ protein levels in the sputum supernatants of both groups were studied by cytokine bead array and are represented (A). Horizontal bars represent median values and IQR. Eosinophils were studied as in figure 3 . The correlation between sputum absolute eosinophil count and TGF- $\beta$ levels is shown (B).
A

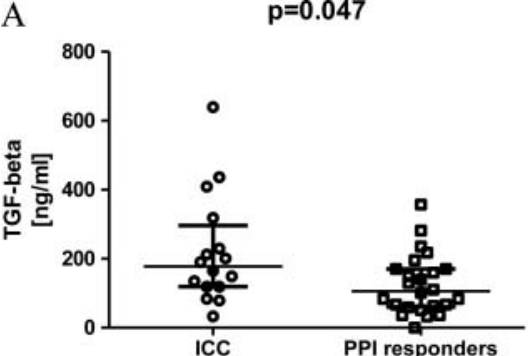

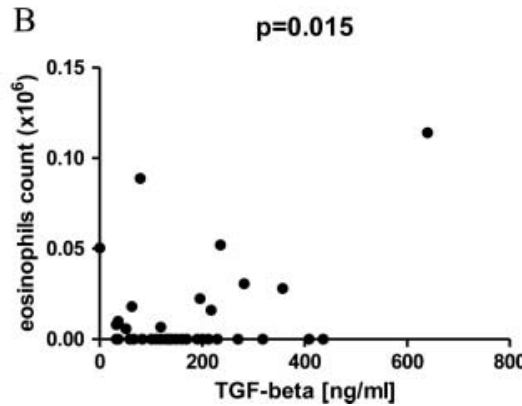

higher sputum MCP-1 protein expression in patients with CC compared to HC. MCP-1 can be released by monocytes, macrophages, smooth muscle cells and fibroblasts but importantly also by airway epithelial cells ${ }^{22}$ and might be an important repair mechanism upon epithelial injury. ${ }^{23}$ MCP-1 was originally thought to mainly recruit monocytes or macrophages, but might also attract neutrophils ${ }^{24}$ towards the site of inflammation. We found a significant linear correlation between sputum MCP-1 levels and sputum neutrophil counts. This could point to an in vivo chemotactic function of MCP-1 but might also be related to its antiapoptotic activity on neutrophils. MCP-1 might furthermore induce the production other proneutrophilic mediators, such as IL-6 and IL-8, for example, by neutrophils (autocrine stimulation). ${ }^{25}$ This could explain why we could also observe a tendency towards increased IL-6 and IL-8 levels in patients with CC. IL-8 by itself might furthermore act as neutrophil attractant and also play its role as an antiapoptotic agent. ${ }^{25}$ Indeed, we also observed a linear correlation between sputum neutrophil counts and sputum IL-8 protein expression levels. It is therefore possible that airway epithelial cells (initially triggered by mechanical stress or pressure) might also increase and maintain their chemokine and cytokine production by autocrine loop, leading to chronic epithelial stimulation and MCP-1 production, hence initiating a self-perpetuating mechanism of epithelial injury, chemokines release and finally neutrophil attraction to the site of inflammation. However, the latter could not be confirmed by recent in vitro resting neutrophil chemotaxis assays. $^{25}$ Despite the observed correlations between sputum neutrophil count and MCP-1 as well as IL-8, and somehow in contrast with the hypothesis of neutrophil recruitment by MCP-1 and/or IL-8, we could not find increased neutrophils in the induced sputum of individuals with CC compared to HC. We furthermore show that a significantly larger proportion of patients with CC express sputum TSLP mRNA when compared to HC. TSLP is produced by epithelial cells as well as dendritic cells ${ }^{26}$ and increased TSLP expression in airway epithelial cells from patients with severe asthma has recently been described. ${ }^{27}$ Our findings suggest that, similarly to MCP-1, TSLP might also play a major role in CC and the clinical availability of a TSLP blocking antibody, certainly stresses the relevance of these findings. TSLP is however not expressed in all patients. Xie and coworkers reported also increased TGF- $\beta$ gene and protein expression in bronchoalveolar lavage (BAL) and airway mucosa from patients with CC compared to healthy subjects. ${ }^{28}$ We were however, not able to confirm their findings in induced sputum samples from the whole group of patients with CC. We observed that in the group of PPI responders, TGF- $\beta$ levels were significantly lower than in patients with ICC. Treatment with PPI might therefore be responsible for the absence of significantly increased TGF- $\beta$ levels in the entire group of patients with CC.

We did not find differences in lymphocyte percentages on cytospin or sputum CD3 $\gamma$ (reflecting infiltrating T cells) between patients with CC and HC. Further on, the expression of Th1/ Th2/Th17/T regulatory (Treg) profile of cytokines is also similar in both groups. We conclude that $\mathrm{T}$ cell cytokines probably do not play a major role in airway inflammation as observed in our CC population. These findings argue against the hypothesis that airway inflammation due to gastric content aspiration could lead to disturbances in the T helper cell balance. ${ }^{6}$

Other studies also indicates that patients with $\mathrm{CC}$ with $\mathrm{RO}$ show increased relative and absolute amounts of sputum eosinophils in comparison to patients with CC without RO. This has been previously reported in GORD-related CC. ${ }^{29}$ Sputum IL-5 and eotaxin-1 mRNA levels, potentially responsible for eosinophil recruitment, however, were not different between our two groups. The observation showing different eosinophil counts between $\mathrm{RO}+$ and $\mathrm{RO}-$ patients with $\mathrm{CC}$, could at least point to the existence of different CC phenotypes, as has been observed in asthma since several years. ${ }^{30}$

Another important clue is the effect of GORD treatment on GORD-related CC. Our finding that TGF- $\beta$ levels are higher in the PPI non-responders (which is not correlated with the duration of CC or subjective impact of CC on QoL) compared to responders, fits with the idea that PPI responders might have other airway inflammatory characteristics. Alternatively, it is of course not impossible that TGF- $\beta$ levels in these patients dropped as a result of treatment, leading to less epithelial stress and hence decreased cytokine production.

We conclude that CC probably is a disease with different disease phenotypes, leading to different inflammatory characteristics depending on the primary underlying trigger or comorbidity. However, high sputum MCP-1 levels are present in a large group of patients with $\mathrm{CC}$ and a large proportion of these patients with CC have increased sputum TSLP levels, most likely produced by airway epithelial cells, as a consequence of direct mechanical stress or reflex-induced epithelial nerve stimulation.

Acknowledgements We would like to thank M Litwa, G Nadobna and K Baraniecka from the Department of Internal Medicine, Geriatrics and Allergology at Wroclaw Medical University, for their help in laboratory part of this study. MG was a recipient of following fellowships: Belgian Thoracic Society fellowship and Flemish Government fellowship. Lieven Dupont and Dominique Bullens are recipients of a senior clinical investigator fellowship from the Fund for Scientific Research Vlaanderen (FWO), Flanders, Belgium. The study was supported by research fellowship within 'Development Program of Wroclaw Medical University' funded from European Social Fund, Human Capital, national Cohesion Strategy' (contract no. UDA-POKL.04.01.01-00-010/08-00). 
Contributors $M G, S S, A D, A K, E D, W B$ recruited patients and were responsible for the experimental data. MG, WM, LJD, BP and DMB were responsible for the study design. MG wrote the manuscript and all authors contributed to the review process.

\section{Competing interests None.}

Patient consent Obtained.

Provenance and peer review Not commissioned; externally peer reviewed.

\section{REFERENCES}

1 Morice A, Fontana GA, Belvisi MG, et al. ERS guidelines on the assessment of cough. Eur Respir J 2007:29:1256-76.

2 Dent J, El-Serag HB, Wallander MA, et al. Epidemiology of gastro-oesophageal reflux disease: a systematic review. Gut 2005;54:710-17.

3 Pavord I, Chung KF. Management of chronic cough. Lancet 2008;371:1375-84.

4 Barnes P. Neurogenic inflammation in the airways. Respir Physiol 2001;125:145-54.

5 Farrell S, McMaster C, Gibson D, et al. Pepsin in bronchoalveolar lavage fluid: a specific and sensitive method of diagnosing gastro-oesophageal reflux-related pulmonary aspiration. J Pediatr Surg 2006;41:289-93.

6 Barbas AS, Downing TE, Balsara KR, et al. Chronic aspiration shifts the immune response from Th1 to Th2 in a murine model of asthma. Eur J Clin Invest 2008:38:596-602.

7 Grabowski M, Kasran A, Seys S, et al. Pepsin and bile acids in induced sputum of chronic cough patients. Respir Med 2011;105:1257-61.

8 Holgate ST. The airway epithelium is central to the pathogenesis of asthma. Allergol Int 2008; 57:1-10.

9 Hammad H, Lambrecht BN. Dendritic cells and epithelial cells: linking innate and adaptive immunity in asthma. Nat Rev Immunol 2008;8:193-204.

10 Nicholas B, Djukanović R. Induced sputum: a window to lung pathology. Biochem Soc Trans 2009;37:868-72.

11 Armstrong $D$, Bennett JR, Blum AL, et al. The endoscopic assessment of esophagitis: a progress report on observer agreement. Gastroenterology 1996;111:85-92.

12 Birring S, Prudon B, Carr AJ, et al. Development of a symptom specific health status measure for patients with chronic cough: Leicester Cough Questionnaire (LCQ). Thorax 2003:58:339-43.

13 Truyen $E$, Coteur L, Dilissen $E$, et al. Evaluation of airway inflammation by quantitative Th1/Th2 cytokine mRNA measurement in sputum of asthma patients. Thorax 2006:61:202-8.

14 Glare EM, Divjak M, Bailey MJ, et al. $\beta$-Actin and GAPDH housekeeping gene expression in asthmatic airways is variable and not suitable for normalising mRNA levels. Thorax 2002;57:765-70.
15 Bullens D, Truyen E, Coteur L, et al. IL-17 mRNA in sputum of asthmatic patients: linking T cell driven inflammation and granulocytic influx? Respir Res 2006;3:135.

16 Giulietti A, Overbergh L, Valckx D, et al. An overview of real-Time Quantitative PCR: applications to quantify cytokine gene expression. Methods 2001;25:386-401.

17 Vanaudenaerde B, De Vleeschauwer SI, Vos R, et al. The role of the IL23/IL17 axis in bronchiolitis obliterans syndrome after lung transplantation. Am J Transplant 2008;8:1911-20

18 Ying $\mathrm{S}, \mathrm{O}^{\prime}$ Connor B, Ratoff J, et al. Thymic stromal lymphopoietin expression is increased in asthmatic airways and correlates with expression of Th2-attracting chemokines and disease severity. J Immunol 2005;174:8183-90.

19 Thomas R, Norman JC, Huynh TT, et al. Mechanical stretch has contrasting effects on mediator release from bronchial epithelial cells, with a rho-kinase-dependent component to the mechanotransduction pathway. Respir Med 2006;100:1588-97.

20 Shibata Y, Nakamura $\mathrm{H}$, Kato $\mathrm{S}$, et al. Cellular detachment and deformation induce IL-8 gene expression in human bronchial epithelial cells. J Immunol 1996;156:772-7

21 Tschumperlin DJ, Shively JD, Kikuchi T, et al. Mechanical stress triggers selective release of fibrotic mediators from bronchial epithelium. Am J Respir Crit Care Med 2003;28:142-9.

22 Huvenne W, Callebaut I, Reekmans K, et al. Staphylococcus aureus enterotoxin B augments granulocyte migration and survival via airway epithelial cell activation. Allergy 2010;65:1013-20.

23 Crosby L, Waters CM. Epithelial repair mechanisms in the lung. Am J Physiol Lung Cell Mol Physiol 2010;298:15-31

24 Johnston B, Burns AR, Suematsu M, et al. Chronic inflammation upregulates chemokine receptors and induces neutrophil migration to monocyte chemoattractant protein-1. J Clin Invest 1999;103:1269-76.

25 Yang $E_{\text {, Choi }}$, Ko J, et al. Differential effect of CCL2 on constitutive neutrophil apoptosis between normal and asthmatic subjects. J Cell Physiol 2012:227:2567-77.

26 Ito T, Liu YJ, Arima K. Cellular and molecular mechanisms of TSLP function in human allergic disorders-TSLP programs the "Th2 code" in dendritic cells. Allergol Int 2012;61:35-43.

27 Shikotra A, Choy DF, Ohri CM, et al. Increased expression of immunoreactive thymic stromal lymphopoietin in patients with severe asthma. J Allergy Clin Immunol 2012;129:104-11.

28 Xie $\mathrm{S}$, Macedo $\mathrm{P}$, Hew M, et al. Expression of transforming growth factor-beta (TGF-beta) in chronic idiopathic cough. Respir Res 2009;22:40.

29 McGarvey L, Forsythe P, Heaney LG, et al. Bronchoalveolar lavage findings in patients with chronic nonproductive cough. Eur Respir J 1999;13:59-65.

30 Gibson PG. Inflammatory phenotypes in adult asthma: clinical applications. Clin Respir J 2009;3:198-206. 